\title{
Factors Affecting Postoperative Sagittal Alignment after Lateral Lumbar Interbody Fusion in Adult Spinal Deformity: Posterior Osteotomy, Anterior Longitudinal Ligament Rupture, and Endplate Injury
}

\author{
Hiroaki Nakashima ${ }^{1}$, Tokumi Kanemura ${ }^{1}$, Kotaro Satake ${ }^{1}$, Yoshimoto Ishikawa ${ }^{1}$, \\ Jun Ouchida ${ }^{1}$, Naoki Segi ${ }^{2}$, Hidetoshi Yamaguchi ${ }^{2}$, Shiro Imagama ${ }^{2}$ \\ ${ }^{1}$ Department of Orthopedic Surgery, Konan Kosei Hospital, Konan, Japan \\ ${ }^{2}$ Department of Orthopedic Surgery, Nagoya University Graduate School of Medicine, Nagoya, Japan
}

\begin{abstract}
Study Design: Prospective cohort study.
Purpose: To identify factors that affect sagittal alignment correction in lateral lumbar interbody fusion (LIF) surgery for adult spinal deformity (ASD) and to investigate the degree of correction in each condition.

Overview of Literature: LIF is a useful procedure for ASD, but the degree of correction can be affected by posterior osteotomy, intraoperative endplate injury, or anterior longitudinal ligament (ALL) rupture.

Methods: Radiographical data for 30 patients who underwent LIF for ASD were examined prospectively. All underwent two-stage surgery (LIF followed by posterior fixation). Radiographical parameters were measured preoperatively, after LIF, and after posterior fixation; these included the segmental lordotic angle, lumbar lordosis (LL), and other sagittal alignment factors.

Results: $\mathrm{LL}$ was corrected from $16.5^{\circ} \pm 16.7^{\circ}$ preoperatively to $33.4^{\circ} \pm 13.8^{\circ}$ after $\mathrm{LIF}(p<0.001)$ and then to $52.1^{\circ} \pm 7.9^{\circ}$ following posterior fixation $(p<0.001)$. At levels where Schwab grade 2 osteotomy was performed, the acquired segmental lordotic angles from the preoperative value to after posterior fixation and from after LIF to after posterior fixation were $19.5^{\circ} \pm 9.2^{\circ}$ and $9.9^{\circ} \pm 3.9^{\circ}$, respectively. On average, $12.4^{\circ}$ more was added than in cases without osteotomy. Endplate injury was identified at 21 levels (19.4\%) after LIF, with a mean loss of $3.4^{\circ}$ in the acquired segmental lordotic angle $\left(5.3^{\circ} \pm 8.4^{\circ}\right.$ and $1.9^{\circ} \pm 5.9^{\circ}$ without and with endplate injury, respectively). ALL rupture was identified at seven levels $(6.5 \%)$, and on average $19.3^{\circ}$ more was added in these cases between the preoperative and postoperative values than in cases without ALL rupture.

Conclusions: LIF provides adequate sagittal alignment restoration for ASD, but the degree of correction is affected by grade 2 osteotomy, intraoperative endplate injury, and ALL rupture.
\end{abstract}

Keywords: Lateral lumbar interbody fusion; Minimally invasive surgical procedures; Segmental sagittal alignment; Adult spinal deformity; Osteotomy; Anterior longitudinal ligament rupture; Endplate injury

Received Oct 22, 2018; Revised Dec 24, 2018; Accepted Dec 30, 2018

Corresponding author: Hiroaki Nakashima

Department of Orthopedic Surgery, Konan Kosei Hospital, 137 Takayamachi Omatsubara, Konan 483-8704, Japan

Tel: +81-587-51-3333, Fax: +81-587-51-3300, E-mail: hirospine@gmail.com 


\section{Introduction}

The primary goal of adult spinal deformity (ASD) surgery is to restore sagittal balance, which is closely associated with pain and disability $[1,2]$. Disk degeneration is the main cause of ASD, and a correction at the interbody space is one of the most appropriate surgical methods $[3,4]$. Lateral lumbar interbody fusion (LIF) is widely used for ASD [5-7]. This technique, which was recently introduced, uses a lateral trans-psoas approach to the disk, which involves less invasive surgical exposure, avoids the major vessels, and permits the placement of large interbody cages that allow the correction of the deformity [6-8].

LIF is effective for the correction of moderate sagittal plane deformities when used in combination with traditional open posterior techniques that use facet osteotomies and a rod cantilever technique. Storm et al. [9] reported that LIF using an open posterior approach for ASD surgery with facet osteotomies and pedicle subtraction osteotomy could restore lumbar lordosis (LL) by $11^{\circ} \pm 7^{\circ}$ and $29^{\circ} \pm 10^{\circ}$, respectively.

Various factors influence this degree of correction in sagittal plane deformity. One of the most important is posterior osteotomy, with the degree of correction increasing with a higher grade of osteotomy. However, osteotomy brings risks of intraoperative massive bleeding and neurological deficit, so cases and levels that need osteotomy should undergo thorough consideration preoperatively. As yet, no detailed data have been reported regarding how the degree of sagittal correction in each intervertebral disc space relates to whether or not osteotomy was performed [10], and the information needed for surgical planning is limited.

In addition to posterior osteotomy, the presence of endplate injury in LIF and anterior longitudinal ligament (ALL) rupture can also affect the degree of sagittal correction [11-18]. Endplate injury is an occasional intraoperative complication. It has been reported to occur at $0.3 \%-22 \%$ of LIF levels, and it can diminish sagittal plane correction [11-14]. If endplate injury is confirmed, the predicted degree of correction should be revised and posterior osteotomy or other procedures reconsidered, depending on the specifics of the case. ALL rupture can also occur accidentally during LIF and posterior correction, which can greatly affect sagittal correction. Anterior column reconstruction has introduced been introduced, and a greater lordotic angle can be obtained by severing the ALL and using a larger angle of cages [15-18].

The purpose of the current study was to investigate the degree of sagittal correction in the segmental intervertebral disc space after posterior osteotomy, endplate injury, or ALL rupture in patients with ASD, and to elucidate the correction angle data needed for surgical planning and modification.

\section{Materials and Methods}

\section{Study design}

This study was approved by our institutional ethics committees (IRB approval no., 29-030 [0315]). We prospectively reviewed radiographical records from consecutive patients with ASD (coronal Cobb angle $>30^{\circ}$ and/or LL $<20^{\circ}$ ) who underwent LIF between 2013 and 2016. We applied the following exclusion criteria: $<20$ years old; $<3$-level involvement; <1-year follow-up; osteotomy equal to or greater than pedicle partial body osteotomy (Schwab classification grade 3) [10]; and the presence of any other disease, such as tumors, traumas, or infections. Finally, 30 patients (mean age, $72.3 \pm 8.0$ years; 6 men, 24 women) were included in this study.

\section{Surgical procedure}

The patients all underwent two-stage surgery. LIF was followed about 1 week later by posterior correction and fusion using bilateral pedicle screw fixation.

The LIF procedures followed the surgical technique described by Ozgur et al. [5], applying extremely lateral lumbar fusion using Coroent cages (NuVasive Inc., San Diego, CA, USA) [19]. For the endplate preparation, the surface was marked with a box cutter to make a soft indentation and an annular incision was then made with a knife. After removing the disk material with a rongeur, a Cobb elevator was advanced gently along the endplates, under fluoroscopy guidance, to release the contralateral annulus. After cage size trials, further disk curettage was performed and the endplates were rasped. The cages were inserted using two containment sliders to protect the endplates and to keep the graft material inside the cage.

For all the cases, the posterior correction and fusion used traditional open posterior techniques with a rod cantilever technique with or without facet osteotomies. The levels for grade 2 osteotomy were determined based on a 
preoperative radiographical evaluation [10].

\section{Radiographical analysis}

Preoperative, post-LIF, and post-posterior fusion whole spine radiographs were obtained with the patient in an upright standing position. The following radiological parameters were measured: segmental lordotic angle (the Cobb angle between the upper and the lower end plates of each fused segment), LL, pelvic tilt (PT), pelvic incidence (PI), and the sagittal vertical axis (SVA).

Endplate injury was assessed using computed tomography (CT) multiplanar reconstruction in contiguous 1-mm slices through the cages, acquired a few days after the LIF. The sagittal plane of each segment was evaluated, and endplate injury was defined as a cage sinking in by $>2 \mathrm{~mm}$ from the vertebral endplate (Fig. 1D).

ALL rupture was assessed using magnetic resonance
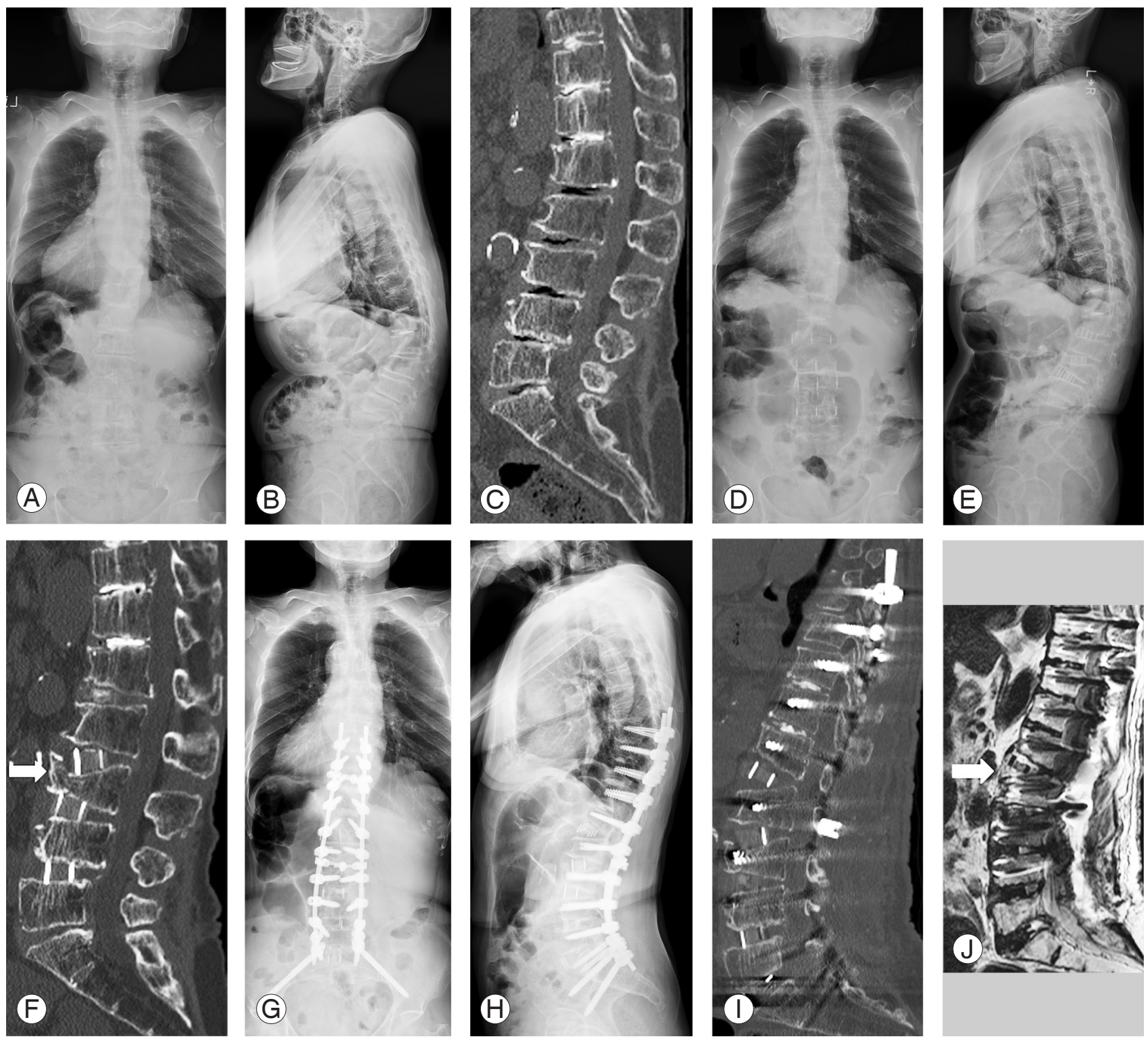

Fig. 1. Images for a representative case of a 78-year-old woman with degenerative kyphosis. (A-C) Preoperative images: whole spine $\mathrm{X}$-rays providing anteroposterior (A) and lateral (B) views; and a sagittal CT image (C). (D-F) Images after lumbar interbody fusion: whole spine X-rays providing anteroposterior (D) and lateral (E) views; and a sagittal CT image (F). A cephalad endplate injury at L3 (white arrow) was identified on postoperative CT (F). (G-I) Postoperative images: whole spine X-rays providing anteroposterior (G) and lateral (H) views; and a sagittal CT image (I). (J) An ALL rupture (white arrow) at L1-2 was identified on magnetic resonance imaging after posterior fixation. The ALL was observed to be incontinuous (white arrow). CT, computed tomography; ALL, anterior longitudinal ligament. 
imaging (MRI) in the sagittal plane after posterior instrumentation and fusion. All patients underwent postoperative MRI within 2 weeks after surgery. ALL rupture was defined by an incontinuous ALL identified on MRI T2weighted images (Fig. 1J).

\section{Statistical analysis}

IBM SPSS Statistics ver. 21.0 (IBM Corp., Armonk, NY, USA) was used for the statistical analysis. Data are presented as mean \pm standard deviation. Student $t$-test and one-way analysis of variance (with Tukey's test for the post hoc analysis) were used to compare two and three groups, respectively. A $p$-value $<0.05$ was considered to indicate a statistically significant difference.

\section{Results}

LIF was performed at a total of 108 interbody levels. The mean fusion level after posterior instrumentation was $7.4 \pm 1.7$ levels. Table 1 summarizes the radiological parameters preoperatively, after LIF, and after posterior fixation. The preoperative mean values of the radiographical parameters were as follows: LL, $16.5^{\circ} \pm 16.7^{\circ}$; SVA, $94.5 \pm 57.5 \mathrm{~mm}$; PI minus LL (PI-LL), $34.4^{\circ} \pm 16.5^{\circ}$; and $\mathrm{PT}, 31.1^{\circ} \pm 10.3^{\circ}$. After LIF but prior to posterior fixation, all the radiographical parameters showed significant improvement: LL, $33.4^{\circ} \pm 13.8^{\circ}(p<0.001)$; SVA, $39.2 \pm 44.7$ $\mathrm{mm}(p<0.001)$; PI-LL, $10.2^{\circ} \pm 17.5^{\circ}(p<0.001)$; and PT, $16.9^{\circ} \pm 15.9^{\circ}(p<0.001)$. In the posterior correction and fixation, Schwab grade 2 osteotomies were performed at seven levels (6.5\%) [10]. After posterior fixation, all the radiographical parameters showed significant improvement compared with before the posterior fixation: LL, $52.1^{\circ} \pm 7.9^{\circ}(p<0.001)$; SVA, $18.2 \pm 36.6 \mathrm{~mm}(p<0.001)$; PILL, $-9.1^{\circ} \pm 16.0^{\circ}(p<0.001)$; and PT, $9.1^{\circ} \pm 17.9^{\circ}(p<0.001)$. After LIF but before posterior fixation, LL and PT had already been corrected to $47.5 \%$ and $64.5 \%$ of their final total extent of correction, respectively.

After LIF, endplate injury was identified at 21 levels on CT (19.4\%). The segmental lordotic angle was $5.3^{\circ} \pm 8.4^{\circ}$ at levels without endplate injury, but $1.9^{\circ} \pm 5.9^{\circ}$ at levels with endplate injury, with a smaller acquired angle $(p=0.08)$. The mean loss in the acquired segmental lordotic angle due to endplate injury was $3.4^{\circ}$.

ALL rupture was identified at seven levels (6.5\%) and grade 2 osteotomy was performed at seven levels (6.5\%). The acquired segmental lordotic angle at levels with ALL rupture from after LIF to after posterior fixation and from the preoperative values to after posterior fixation were $14.1^{\circ} \pm 6.2^{\circ}$ and $26.4^{\circ} \pm 9.2^{\circ}$, respectively (Table 2). At levels without ALL rupture or grade 2 osteotomy, the acquired segmental lordotic angle from after LIF to after posterior fixation and from the preoperative values to after posterior fixation were $3.1^{\circ} \pm 5.7^{\circ}$ and $7.1^{\circ} \pm 9.1^{\circ}$, respectively. Of note, these acquired segmental lordotic angles were sig-

Table1. Pre- and postoperative spinal alignment

\begin{tabular}{lccc} 
Variable & Preoperative & After lateral lumbar interbody fusion, prior to posterior fixation & Postoperative \\
$\mathrm{LL}\left({ }^{\circ}\right)$ & $16.5 \pm 16.7$ & $33.4 \pm 13.8$ & $52.1 \pm 7.9$ \\
\hline Sagittal vertical axis $(\mathrm{mm})$ & $94.5 \pm 57.5$ & $9.2 \pm 44.7$ & $18.2 \pm 36.6$ \\
\hline Pelvic incidence- $\mathrm{LL}\left({ }^{\circ}\right)$ & $34.4 \pm 16.5$ & $10.2 \pm 17.5$ & $-9.1 \pm 16.0$ \\
\hline Pelvic tilt $\left(^{\circ}\right)$ & $31.1 \pm 10.3$ & $16.9 \pm 15.9$ & $9.1 \pm 17.9$ \\
\hline
\end{tabular}

$\mathrm{LL}$, lumbar lordosis.

Table 2. The acquired segmental lordotic angle

\begin{tabular}{|c|c|c|c|c|}
\hline Variable & $\begin{array}{l}\text { No grade } 2 \text { osteotomy } \\
\text { nor ALL rupture }\end{array}$ & Grade 2 osteotomy & ALL rupture & $p$-value \\
\hline Levels $(\%)$ & $94(87.0)$ & $7(6.5)$ & $7(6.5)$ & \\
\hline From lateral lumbar interbody fusion to posterior fixation $\left({ }^{\circ}\right)$ & $3.1 \pm 5.7$ & $9.9 \pm 3.9$ & $14.1 \pm 6.2$ & $<0.0001$ \\
\hline From preoperative to posterior fixation $\left(^{\circ}\right)$ & $7.1 \pm 9.1$ & $19.5 \pm 9.2$ & $26.4 \pm 9.2$ & $<0.0001$ \\
\hline
\end{tabular}

Values are presented as number (\%) or mean \pm standard deviation. Statistical test: one-way analysis of variance (post-hoc Tukey).

$\mathrm{ALL}$, anterior longitudinal ligament. 
nificantly larger at levels with ALL rupture than at those without ALL rupture $(p<0.0001)$; and when there was accidental ALL rupture, an average of $19.3^{\circ}$ more was added from the preoperative to the postoperative stage than in cases without ALL rupture.

At seven levels with complete facet joint osteotomy (Schwab grade 2) [10], the mean acquired segmental lordotic angles from after LIF to after posterior fixation and from the preoperative values to after posterior fixation were $9.9^{\circ} \pm 3.9^{\circ}$ and $19.5^{\circ} \pm 9.2^{\circ}$, respectively (Table 2 ). These acquired angles were significantly larger at levels that underwent grade 2 osteotomy than at levels without ALL rupture or grade 2 osteotomy $(p<0.0001)$ : by adding grade 2 osteotomy, an average of $12.4^{\circ}$ more was added between the preoperative and postoperative stages than in cases without osteotomy.

\section{Representative case}

The technique can be illustrated with a representative case, of a 78-year-old woman with degenerative kyphosis. Her preoperative radiological parameters were as follows: LL, $1^{\circ}$; PT, $31^{\circ}$; PI-LL, $36^{\circ}$; and SVA, $5.5 \mathrm{~cm}$ (Fig. 1A-C). We performed LIF at four levels from L1-2 to L4-5. Postoperative CT identified cephalad endplate injury at L3 (Fig. 1D, E). After LIF, the radiological parameters were as follows: LL, $9^{\circ}$; PT, $26^{\circ}$; and SVA, $2.5 \mathrm{~cm}$. The mean acquired segmental lordotic angle at L1-2, L3-4, and L4-5 was $4.7^{\circ}$; the angle was smaller $\left(2.1^{\circ}\right)$ at $\mathrm{L} 2-3$, where the endplate injury was identified (Fig. 1F). We performed posterior fixation from T9 to the pelvis and L5-S posterior LIF, but not grade 2 osteotomy (Fig. 1G, H). An ALL rupture at L1-2 was identified on MRI after posterior fixation (Fig. 1I, J). The mean acquired segmental lordotic angle at L23, L3-4, and L4-5, from after LIF to after posterior fixation was $7.8^{\circ}$; the angle was larger $\left(13.6^{\circ}\right)$ at L1-2, where the ALL rupture was identified. Postoperatively, the patient's radiological parameters were as follows: $\mathrm{LL}, 60^{\circ}$; PT, $7^{\circ}$; and SVA, $2.8 \mathrm{~cm}$. To prevent anterior cage extrusion, the patient wore a hard brace for 3 months postoperatively. At 1-year follow-up, bony fusion had been achieved in the posterior part of the spine.

\section{Discussion}

This study investigated the factors that affect postoperative sagittal alignment after LIF in patients with ASD. These were found to be grade 2 osteotomy, endplate injury, and ALL rupture. Our results indicated that LIF resulted in a mean increase of $5^{\circ}$ in the segmental lordotic angle compared with the preoperative angle, and that there was a total increase of $7^{\circ}$ by the postoperative stage, even when grade 2 osteotomy was not performed. In cases where grade 2 osteotomy was performed, the mean acquired lordotic angle was approximately $20^{\circ}$, with $12^{\circ}$ was added to the segmental lordotic angle. In cases where ALL ruptured intraoperatively, the total acquired segmental lordotic angle was $26^{\circ}$, and the acquired lordotic angle increased. Thus, LIF provided adequate sagittal alignment restoration in ASD, but the degree of correction was greatly affected by grade 2 osteotomy, intraoperative endplate injury, and the occurrence of ALL rupture.

LIF was developed in the late 1990s as a minimally invasive fusion technique [5]. The LIF approach enables sufficient disk space preparation and the placement of a large interbody cage from the lateral side of the spine $[5,20]$. This cage is useful for the correction of ASD to restore disk height in cases where degeneration is the main cause of the deformity [3]. In previous studies, LIF was shown to have improved segmental sagittal alignment [21], with an increase in disk height of $41.9 \%-83 \%$ and an increase in foraminal height of $13.5 \%-83 \%$ [22,23]. Anand et al. [23] reported an $8.1^{\circ}$ increase in segmental lordosis in ASD cases treated with LIF using a $10^{\circ}$ cage. In an investigation of 80 patients (mean fusion level, 1.5 levels), Shiga et al. [24] found that the acquired lordotic angle was $3.8^{\circ}$ after LIF with $6^{\circ}$ lordotic cages. In the present study, LIF was found to result in mean increase in segmental lordotic angle of $5^{\circ}$ compared with the preoperative angle, with a total increase of $7^{\circ}$ by the postoperative stage; these results were largely consistent with those reported previously.

There is currently no consensus regarding the segmental lordotic angle in cases with additional grade 2 osteotomy, ALL rupture, or intraoperative endplate injury. Grade 2 osteotomy was introduced by Smith-Petersen et al. [25] and elaborated by Geck et al. [26] in their discussions of the use of wide segmental osteotomies and posterior compression along unfused regions of the kyphotic deformity. Grade 2 osteotomy involves the removal of the posterior ligaments, spinous process, and facets to achieve sagittal plane realignment. The degree of kyphotic correction achievable with this technique has been reported to be in the range $9.3^{\circ}-10.7^{\circ}$ per level $[26,27]$. In the present study, grade 2 osteotomy added $12^{\circ}$ to the segmental lor- 
dosis at levels; this was slightly greater than that achieved previously with LIF surgery for ASD. This was because we were able to place a large cage in the anterior part of the vertebral body with LIF, which may have resulted in a slightly larger acquired lordotic angle when closing the posterior part of the spine using osteotomy. Grade 2 osteotomy is a relatively safe and rapid procedure for the correction of gradual kyphosis or hypolordosis [26,27], with less blood loss, a shorter operative time, and fewer neurological complications than with grade 3 osteotomy (pedicle subtraction osteotomy) [28]. However, the blood loss is greater than with grade 1 osteotomy, so the levels at which grade 2 osteotomy is performed should be carefully selected. Although LIF with grade 2 osteotomy can result in a segmental lordotic angle of approximately $20^{\circ}$ in total, the procedures are not suitable in cases with sharp, angular kyphosis; grade 3 osteotomy should be considered in such cases.

Postoperative cage subsidence in LIF surgery has been reported, with a reported incidence of $0.3 \%-22 \%$ of cases [12-14]. Two types of cage subsidence have been identified: one as a result of intraoperative endplate injury, the other appearing gradually during the postoperative period. Intraoperative endplate injury may be highly associated with the progressive settling of cages and with less clinical improvement. In a study that focused specifically on intraoperative endplate injury, Satake et al. [11] reported that $10.4 \%$ of 102 consecutive cases showed signs of intraoperative endplate injury. In that study, the risk factors for endplate injury included female sex, lower bone mineral density, a higher rate of polyetheretherketone used as cage material, and greater cage height [11]. Age was also a factor; ASD is often performed for elderly patients, so the possibility of endplate injury is high. However, there have been no reports that have investigated the incidence of endplate injury in ASD surgery or the loss of acquired lordosis in cases with endplate injury. In the present study, the incidence of endplate injury was $19.4 \%$. The acquired segmental lordotic angle was approximately $2^{\circ}$ in levels with endplate injury, with a mean loss of $3^{\circ}$ in the acquired segmental lordotic angle. Sufficient anterior disc height restoration could not be obtained at levels with endplate injury, making it difficult to achieve sufficient segmental lordotic angle without performing posterior column shortening.

Anterior column reconstruction has recently been introduced to address intraoperative ALL rupture [15-18], and the relationship between severing the ALL and the acquired lordotic angle is attracting considerable attention. Anterior column reconstruction surgery achieves greater segmental correction by severing the ALL, allowing the placement of hyperlordotic cages $[18,29,30]$. A mean segmental lordosis correction of $10^{\circ}-27^{\circ}$ has been reported with anterior column reconstruction using hyperlordotic cages [15-18]. In the present study, the acquired segmental lordotic angle after accidental ALL rupture without hyperlordotic cages was $26^{\circ}$, similar to the previously reported values. The ALL ruptures in the present study occurred during posterior surgery while using a rod cantilever technique for kyphosis correction; they were not due to directly severing the ALL during anterior surgery. This complication might be the result of performing the kyphosis correction by anterior stretching using the rod cantilever technique without posterior shortening. If LIF cages placed in the anterior intervertebral space become unstable after ALL rupture, revision surgery may be needed; spine surgeons should therefore exercise considerable caution.

This study had two limitations. First, the number of cases was relatively small. A prospective large-scale study is therefore needed to validate the results. Second, the diagnostic accuracy of ALL rupture on MRI was unclear. There may have been inaccuracy due to surrounding acute postoperative changes.

\section{Conclusions}

LIF resulted, on average, in a segmental lordotic angle of $5^{\circ}$, with this angle increasing to $12^{\circ}$ when grade 2 osteotomy was also performed. Endplate injury and ALL rupture are not infrequent complications in ASD surgery using LIF; spine surgeons should take care to prevent these complications, which can seriously affect the postoperative sagittal alignment.

\section{Conflict of Interest}

No potential conflict of interest relevant to this article was reported. Tokumi Kanemura is a consultant of Medtronic and Nuvasive.

\section{Author Contributions}

Nakashima H: corresponding author, conception, design, 
and analysis; Nakashima H, Kanmemura T, Satake K, Ishikawa Y, Ouchida J, Segi N, Yamaguchi H: acquisition of data; and Kanemura T, Satake K, and Imagama S: critically revising the article.

\section{References}

1. Glassman SD, Bridwell K, Dimar JR, Horton W, Berven S, Schwab F. The impact of positive sagittal balance in adult spinal deformity. Spine (Phila Pa 1976) 2005;30:2024-9.

2. Daubs MD, Lenke LG, Bridwell KH, et al. Does correction of preoperative coronal imbalance make a difference in outcomes of adult patients with deformity? Spine (Phila Pa 1976) 2013;38:476-83.

3. Youssef JA, Orndorff DO, Patty CA, et al. Current status of adult spinal deformity. Global Spine J 2013;3:51-62.

4. Pritchett JW, Bortel DT. Degenerative symptomatic lumbar scoliosis. Spine (Phila Pa 1976) 1993;18:7003.

5. Ozgur BM, Aryan HE, Pimenta L, Taylor WR. Extreme lateral interbody fusion (XLIF): a novel surgical technique for anterior lumbar interbody fusion. Spine J 2006;6:435-43.

6. Acosta FL, Liu J, Slimack N, Moller D, Fessler R, Koski T. Changes in coronal and sagittal plane alignment following minimally invasive direct lateral interbody fusion for the treatment of degenerative lumbar disease in adults: a radiographic study. J Neurosurg Spine 2011;15:92-6.

7. Bae J, Theologis AA, Strom R, et al. Comparative analysis of 3 surgical strategies for adult spinal deformity with mild to moderate sagittal imbalance. J Neurosurg Spine 2018;28:40-9.

8. Berjano P, Lamartina C. Far lateral approaches (XLIF) in adult scoliosis. Eur Spine J 2013;22 Suppl 2:S24253.

9. Strom RG, Bae J, Mizutani J, Valone F 3rd, Ames CP, Deviren V. Lateral interbody fusion combined with open posterior surgery for adult spinal deformity. J Neurosurg Spine 2016;25:697-705.

10. Schwab F, Blondel B, Chay E, et al. The comprehensive anatomical spinal osteotomy classification. Neurosurgery 2014;74:112-20.

11. Satake K, Kanemura T, Nakashima H, Yamaguchi H, Segi N, Ouchida J. Cage subsidence in lateral inter- body fusion with transpsoas approach: intraoperative endplate injury or late-onset settling. Spine Surg Relat Res 2017;1:203-10.

12. Marchi L, Abdala N, Oliveira L, Amaral R, Coutinho E, Pimenta L. Radiographic and clinical evaluation of cage subsidence after stand-alone lateral interbody fusion. J Neurosurg Spine 2013;19:110-8.

13. Le TV, Baaj AA, Dakwar E, et al. Subsidence of polyetheretherketone intervertebral cages in minimally invasive lateral retroperitoneal transpsoas lumbar interbody fusion. Spine (Phila Pa 1976) 2012;37:126873.

14. Malham GM, Ellis NJ, Parker RM, Seex KA. Clinical outcome and fusion rates after the first 30 extreme lateral interbody fusions. ScientificWorldJournal 2012;2012:246989.

15. Akbarnia BA, Mundis GM Jr, Moazzaz P, et al. Anterior column realignment (ACR) for focal kyphotic spinal deformity using a lateral transpsoas approach and ALL release. J Spinal Disord Tech 2014;27:29-39.

16. Hosseini P, Mundis GM Jr, Eastlack RK, et al. Preliminary results of anterior lumbar interbody fusion, anterior column realignment for the treatment of sagittal malalignment. Neurosurg Focus 2017;43:E6.

17. Mundis GM Jr, Turner JD, Kabirian N, et al. Anterior column realignment has similar results to pedicle subtraction osteotomy in treating adults with sagittal plane deformity. World Neurosurg 2017;105:249-56.

18. Saigal R, Mundis GM Jr, Eastlack R, Uribe JS, Phillips FM, Akbarnia BA. Anterior column realignment (ACR) in adult sagittal deformity correction: technique and review of the literature. Spine (Phila $\mathrm{Pa}$ 1976) 2016;41 Suppl 8:S66-73.

19. Satake K, Kanemura T, Yamaguchi H, Segi N, Ouchida J. Predisposing factors for intraoperative endplate injury of extreme lateral interbody fusion. Asian Spine J 2016;10:907-14.

20. Pimenta L, Turner AW, Dooley ZA, Parikh RD, Peterson MD. Biomechanics of lateral interbody spacers: going wider for going stiffer. ScientificWorldJournal 2012;2012:381814.

21. Costanzo G, Zoccali C, Maykowski P, Walter CM, Skoch J, Baaj AA. The role of minimally invasive lateral lumbar interbody fusion in sagittal balance correction and spinal deformity. Eur Spine J 2014;23 Suppl 6:699-704.

22. Alimi M, Hofstetter CP, Cong GT, et al. Radiological 
and clinical outcomes following extreme lateral interbody fusion. J Neurosurg Spine 2014;20:623-35.

23. Anand N, Cohen RB, Cohen J, Kahndehroo B, Kahwaty S, Baron E. The influence of lordotic cages on creating sagittal balance in the CMIS treatment of adult spinal deformity. Int J Spine Surg 2017;11:23.

24. Shiga Y, Orita S, Inage K, et al. Evaluation of the location of intervertebral cages during oblique lateral interbody fusion surgery to achieve sagittal correction. Spine Surg Relat Res 2017;1:197-202.

25. Smith-Petersen MN, Larson CB, Aufranc OE. Osteotomy of the spine for correction of flexion deformity in rheumatoid arthritis. Clin Orthop Relat Res 1969;66:6-9.

26. Geck MJ, Macagno A, Ponte A, Shufflebarger HL. The Ponte procedure: posterior only treatment of Scheuermann's kyphosis using segmental posterior shortening and pedicle screw instrumentation. J Spinal Disord Tech 2007;20:586-93.
27. Cho KJ, Bridwell KH, Lenke LG, Berra A, Baldus C. Comparison of Smith-Petersen versus pedicle subtraction osteotomy for the correction of fixed sagittal imbalance. Spine (Phila Pa 1976) 2005;30:2030-7.

28. Kim HJ, Piyaskulkaew C, Riew KD. Comparison of Smith-Petersen osteotomy versus pedicle subtraction osteotomy versus anterior-posterior osteotomy types for the correction of cervical spine deformities. Spine (Phila Pa 1976) 2015;40:143-6.

29. Pimenta L, Fortti F, Oliveira L, et al. Anterior column realignment following lateral interbody fusion for sagittal deformity correction. Eur J Orthop Surg Traumatol 2015;25 Suppl 1:S29-33.

30. Uribe JS, Smith DA, Dakwar E, et al. Lordosis restoration after anterior longitudinal ligament release and placement of lateral hyperlordotic interbody cages during the minimally invasive lateral transpsoas approach: a radiographic study in cadavers. J Neurosurg Spine 2012;17:476-85. 